\title{
Ratio asymptotics for multiple orthogonal polynomials
}

\author{
Walter Van Assche
}

\begin{abstract}
We give the asymptotic behavior of the ratio of two neighboring multiple orthogonal polynomials under the condition that the recurrence coefficients in the nearest neighbor recurrence relations converge.
\end{abstract}

\section{Introduction}

Multiple orthogonal polynomials (or Hermite-Padé polynomials) are monic polynomials $P_{\vec{n}}$ with a multi-index $n=\left(n_{1}, \ldots, n_{r}\right) \in \mathbb{N}^{r}$ and of degree $|\vec{n}|=$ $n_{1}+\cdots+n_{r}$ satisfying the orthogonality relations

$$
\int P_{\vec{n}}(x) x^{k} d \mu_{j}(x)=0, \quad k=0,1, \ldots, n_{j}-1,1 \leq j \leq r,
$$

for a system of $r$ positive measures $\left(\mu_{1}, \ldots, \mu_{r}\right)$ on the real line. They satisfy a system of nearest neighbor recurrence relations $[\mathbf{1 3}, \S 23.1 .4][\mathbf{1 7}]$

$$
x P_{\vec{n}}(x)=P_{\vec{n}+\vec{e}_{k}}(x)+b_{\vec{n}, k} P_{\vec{n}}(x)+\sum_{j=1}^{r} a_{\vec{n}, j} P_{\vec{n}-\vec{e}_{j}}(x),
$$

for $1 \leq k \leq r$. We will assume that the recurrence coefficients satisfy the following. Let $n_{j}=\left\lfloor q_{j} n\right\rfloor$, where $q_{j}>0$ and $\sum_{j=1}^{r} q_{j}=1$, so that $|\vec{n}| / n \rightarrow 1$ as $n \rightarrow \infty$. We say that multiple orthogonal polynomials belong to the class $M(\vec{a}, \vec{b})$ if

$$
\lim _{n \rightarrow \infty} a_{\vec{n}, j}=a_{j}, \quad \lim _{n \rightarrow \infty} b_{\vec{n}, j}=b_{j} .
$$

Observe that the limits $\vec{a}=\left(a_{1}, \ldots, a_{r}\right)$ and $\vec{b}=\left(b_{1}, \ldots, b_{r}\right)$ depend on $\vec{q}=$ $\left(q_{1}, \ldots, q_{r}\right)$ which indicate the direction in $\mathbb{N}^{r}$ in which the multi-index $\vec{n}$ tends to infinity. Our interest is to obtain the asymptotic behavior of the ratio $P_{\vec{n}+\vec{e}_{k}}(x) / P_{\vec{n}}(x)$ of two neighboring multiple orthogonal polynomials. The main result is

TheOREM 1.1. Suppose the multiple orthogonal polynomials $P_{\vec{n}}$ and $P_{\vec{n}+\vec{e}_{k}}$ have interlacing real zeros for every $\vec{n}$ and $1 \leq k \leq r$ and the recurrence coefficients have asymptotic behavior given by (1.2) uniformly in a closed neighborhood of $\vec{q}=$

2010 Mathematics Subject Classification. Primary 42 C05.

Supported by KU Leuven research grant OT/12/073, FWO research project G.0934.13, and the Belgian Interuniversity Attraction Poles programme P7/18. 
$\left(q_{1}, \ldots, q_{r}\right)$, where $n_{j}=\left\lfloor q_{j} n\right\rfloor$, with $q_{j}>0$ and $\sum_{j=1}^{r} q_{j}=1$, and $b_{i} \neq b_{j}$ whenever $i \neq j$. Then

$$
\lim _{n \rightarrow \infty} \frac{P_{\vec{n}+\vec{e}_{k}}(x)}{P_{\vec{n}}(x)}=z(x)-b_{k}
$$

uniformly on compact subsets $K$ of $\mathbb{C} \backslash \mathbb{R}$, where $z$ is the solution of the algebraic equation

$$
(z-x) B_{r}(z)+A_{r-1}(z)=0
$$

for which $z(x)-x \rightarrow 0$ when $x \rightarrow \infty$, where $B_{r}(z)=\left(z-b_{1}\right)\left(z-b_{2}\right) \cdots\left(z-b_{r}\right)$ and $A_{r-1}$ is the polynomial of degree $r-1$ for which

$$
\frac{A_{r-1}(z)}{B_{r}(z)}=\sum_{j=1}^{r} \frac{a_{j}}{z-b_{j}} .
$$

The condition that $P_{\vec{n}}$ and $P_{\vec{n}+\vec{e}_{k}}$ have interlacing real zeros for every $\vec{n}$ and $1 \leq k \leq r$ is not easy to check, but there are sufficient conditions that give this interlacing property. For instance, if $a_{\vec{n}, j}>0$ for every $\vec{n}$ for which $n_{j}>0$, then the interlacing property holds $[\mathbf{1 1}$, Thm. 2.2].

If the recurrence coefficients are unbounded, then one can investigate the ratio of two neighboring multiple orthogonal polynomials in which the variable is scaled, taking into account the growth of the recurrence coefficients.

THEOREM 1.2. Suppose the multiple orthogonal polynomials $P_{\vec{n}}$ and $P_{\vec{n}+\vec{e}_{k}}$ have interlacing real zeros for every $\vec{n}$ and $1 \leq k \leq r$ and that for some $\gamma>0$

$$
\lim _{n \rightarrow \infty} \frac{a_{\vec{n}, j}}{n^{2 \gamma}}=a_{j}, \quad \lim _{n \rightarrow \infty} \frac{b_{\vec{n}, j}}{n^{\gamma}}=b_{j},
$$

uniformly in a closed neigborhood of $\vec{q}=\left(q_{1}, \ldots, q_{r}\right)$, where $\vec{n}=\left(\left\lfloor q_{1} n\right\rfloor, \ldots,\left\lfloor q_{r} n\right\rfloor\right)$, with $q_{j}>0$ and $\sum_{j=1}^{r} q_{j}=1$, and $b_{i} \neq b_{j}$ whenever $i \neq j$. Then

$$
\lim _{n \rightarrow \infty} \frac{P_{\vec{n}+\vec{e}_{k}}\left(n^{\gamma} x\right)}{n^{\gamma} P_{\vec{n}}\left(n^{\gamma} x\right)}=z(x)-b_{k}
$$

uniformly on compact subsets $K$ of $\mathbb{C} \backslash \mathbb{R}$, where $z$ is the solution of the algebraic equation

$$
(z-x) B_{r}(z)+A_{r-1}(z)=0
$$

for which $z(x)-x \rightarrow 0$ when $x \rightarrow \infty$, where $B_{r}(z)=\left(z-b_{1}\right)\left(z-b_{2}\right) \cdots\left(z-b_{r}\right)$ and $A_{r-1}$ is the polynomial of degree $r-1$ for which

$$
\frac{A_{r-1}(z)}{B_{r}(z)}=\sum_{j=1}^{r} \frac{a_{j}}{z-b_{j}} .
$$

The multiple orthogonal polynomials $\left(Q_{m}\right)_{m \in \mathbb{N}}$ on the stepline, i.e., for $m=$ $k r+j$ we set $\vec{n}_{m}=(k+1, \ldots, k+1, k, \ldots, k)$, with $j$ times $k+1$ and $r-j$ times $k$, and $Q_{m}=P_{\vec{n}_{m}}$, satisfy a higher order recurrence relation of the form

$$
x Q_{m}(x)=Q_{m+1}(x)+\sum_{k=0}^{r} \beta_{m, k} Q_{m-k}(x),
$$

and many authors investigated the multiple orthogonal polynomials on the stepline and the recurrence coefficients $\beta_{m, k}$. Kalyagin [14] showed that for Angelesco systems (i.e., the measures $\mu_{j}$ are supported on pairwise disjoint intervals $\Delta_{j}$ ) the 
recurrence coefficients $\left(\beta_{m, k}\right)_{m}$ are asymptotically periodic for $0 \leq k \leq r$ and he investigated the asymptotic behavior of the polynomials (assuming a Szegó condition for each $\mu_{j}$ on $\Delta_{j}$ ) and some ratio asymptotics. Aptekarev, Kalyagin and Saff $[\mathbf{3}]$ investigated the ratio asymptotics of the stepline polynomials when $\beta_{m, k}=0$ for $0 \leq k \leq r-1$ and $\lim _{m \rightarrow \infty} \beta_{m, r}=b$. Aptekarev et al. [2] showed that the recurrence coefficients $\left(\beta_{m, k}\right)_{m}$ are asymptotically periodic for Nikishin systems, and the ratio of neighboring multiple orthogonal polynomials for Nikishin systems was investigated in $[\mathbf{4}],[\mathbf{1 5}]$ and $[\mathbf{1 0}]$. In this paper we are interested in the ratio asymptotics of multiple orthogonal polynomials when the multi-index tends to infinity in any direction in $\mathbb{N}^{r}$. The recurrence relation for the stepline polynomials only gives asymptotics for the multiple orthogonal polynomials near the diagonal, i.e., the case when $q_{j}=1 / r$ for $1 \leq j \leq r$. The nearest neighbor recurrence relations allow us to investigate the asymptotic behavior in any direction. Our Theorems 1.1 and 1.2 show that, under the conditions that we impose, the ratio asymptotics for two neighboring multiple orthogonal polynomials is given in terms of an algebraic function $z(x)$ which is the solution of an algebraic equation (1.3) of order $r+1$. The Riemann surface for this algebraic function has genus 0 since for given $z$ one can find $x$ in a unique way from (1.3). We illustrate our results using some known systems of multiple orthogonal polynomials in Section 5 .

\section{Proof of Theorem 1.1}

Use the recurrence relation (1.1) and divide by $P_{\vec{n}}(x)$ to find

$$
x=\frac{P_{\vec{n}+\vec{e}_{k}}(x)}{P_{\vec{n}}(x)}+b_{\vec{n}, k}+\sum_{j=1}^{r} a_{\vec{n}, j} \frac{P_{\vec{n}-\vec{e}_{j}}(x)}{P_{\vec{n}}(x)} .
$$

The interlacing of the zeros of $P_{\vec{n}}$ and $P_{\vec{n}-\vec{e}_{j}}$ implies the partial fractions decomposition

$$
\frac{P_{\vec{n}-\vec{e}_{j}}(x)}{P_{\vec{n}}(x)}=\sum_{k=1}^{|\vec{n}|} \frac{A_{\vec{n}, k}}{x-x_{\vec{n}, k}},
$$

with positive residues $A_{\vec{n}, k}>0$, where $\left\{x_{\vec{n}, k}, 1 \leq k \leq|\vec{n}|\right\}$ are the zeros of $P_{\vec{n}}$. Furthermore, since all our multiple orthogonal polynomials are monic, we have

$$
\sum_{k=1}^{|\vec{n}|} A_{\vec{n}, k}=1 .
$$

Hence for $x \in K \subset \mathbb{C} \backslash \mathbb{R}$ we have the bound

$$
\left|\frac{P_{\vec{n}-\vec{e}_{j}}(x)}{P_{\vec{n}}(x)}\right| \leq \sum_{k=1}^{|\vec{n}|} \frac{A_{\vec{n}, k}}{\left|x-x_{\vec{n}, k}\right|} \leq \frac{1}{\delta},
$$

where $\delta=\min \{|x-y|: x \in K, y \in \mathbb{R}\}>0$ is the minimal distance between $K$ and $\mathbb{R}$. Hence $\left\{P_{\vec{n}-\vec{e}_{j}}(x) / P_{\vec{n}}(x): \vec{n} \in \mathbb{N}^{r}\right\}$ is a normal family on every compact $K \subset \mathbb{C} \backslash \mathbb{R}$, and by Montel's theorem there exists a subsequence $\left(n_{k}\right)_{k \in \mathbb{N}}$ so that $P_{\vec{n}_{k}-\vec{e}_{j}}(x) / P_{\vec{n}_{k}}(x)$ converges uniformly on $K$, where $\vec{n}_{k}=\left(\left\lfloor q_{1} n_{k}\right\rfloor, \ldots,\left\lfloor q_{r} n_{k}\right\rfloor\right)$. By taking a further subsequence we can conclude that there is a subsequence $\left(n_{k}\right)_{k \in \mathbb{N}}$ such that

$$
\lim _{k \rightarrow \infty} \frac{P_{\vec{n}_{k}-\vec{e}_{j}}(x)}{P_{\vec{n}_{k}}(x)}=h_{j}(x)
$$


uniformly on $K$ for every $j$ with $1 \leq j \leq r$. Clearly $h_{j}$ is analytic on $K$ and $h_{j}(x) \rightarrow 0$ when $x \rightarrow \infty$. From Lemma 3.3 in Section 3 we find that along this subsequence we also have

$$
\lim _{k \rightarrow \infty} \frac{P_{\vec{n}_{k}+\vec{e}_{j}}(x)}{P_{\vec{n}_{k}}(x)}=\frac{1}{h_{j}(x)},
$$

uniformly on $K$. Hence if we take the limit along this subsequence in (2.1) and use (1.2), then

$$
x=\frac{1}{h_{k}(x)}+b_{k}+\sum_{j=1}^{r} a_{j} h_{j}(x) .
$$

Define $z=z(x)$ by

$$
z=x-\sum_{j=1}^{r} a_{j} h_{j}(x)
$$

then we find that

$$
h_{k}(x)=\frac{1}{z-b_{k}}, \quad 1 \leq k \leq r,
$$

so that (2.3) implies

$$
x-z=\sum_{j=1}^{r} \frac{a_{j}}{z-b_{j}}=\frac{A_{r-1}(z)}{B_{r}(z)},
$$

which gives the algebraic equation (1.3). For $x \rightarrow \infty$ we either have that $z(x)$ remains bounded or $z(x)$ is unbounded. In the latter case

$$
\lim _{x \rightarrow \infty} \frac{A_{r-1}(z)}{B_{r}(z)}=0
$$

so that $z(x)-x \rightarrow 0$, which is the solution that we want. If $z(x)$ remains bounded, then $A_{r-1}(z) / B_{r}(z)$ is unbounded as $x \rightarrow \infty$, which is only possible when $z(x) \rightarrow$ $b_{j}$. This gives $r$ other solutions of the algebraic equation (1.3). Hence along the subsequence $\left(n_{k}\right)_{k \in \mathbb{N}}$ we found the limits $1 / h_{j}(x)=z(x)-b_{j}$ for $1 \leq j \leq r$. The solution $z$ is independent of the subsequence $\left(n_{k}\right)_{k \in \mathbb{N}}$, hence every converging subsequence gives the same limit functions, which implies that the full sequence converges.

\section{Some technical lemmas}

An important step in the proof is that $P_{\vec{n}-\vec{e}_{j}}(x) / P_{\vec{n}}(x)$ and $P_{\vec{n}}(x) / P_{\vec{n}+\vec{e}_{j}}(x)$ have the same limit as $n \rightarrow \infty$. To prove this, one needs some extra results.

Lemma 3.1. Suppose $\left\{D_{\vec{n}}, \vec{n} \in \mathbb{N}^{r}\right\}$ are positive quantities, with $D_{\vec{n}}=0$ whenever $n_{j}<0$ for some $j \in\{1, \ldots, r\}$, satisfying

$$
D_{\vec{n}} \leq A_{\vec{n}}+\sum_{j=1}^{r} a_{j} D_{\vec{n}-\vec{e}_{j}},
$$

with given quantities $A_{\vec{n}}>0$ and $a_{j}>0(1 \leq j \leq r)$. Then

$$
D_{\vec{n}} \leq \sum_{\vec{m} \leq \vec{n}} A_{\vec{n}-\vec{m}}\left(\begin{array}{c}
|\vec{m}| \\
m_{1}, \ldots, m_{r}
\end{array}\right) a_{1}^{m_{1}} \cdots a_{r}^{m_{r}} .
$$


A special case is when $A_{\vec{n}}=A$ for every $\vec{n}$, in which case one has

$$
D_{\vec{n}} \leq A \sum_{k=0}^{|\vec{n}|}\left(\sum_{j=1}^{r} a_{j}\right)^{k}
$$

Proof. We will use induction on the length $|\vec{n}|$. For $|\vec{n}|=0$ we have $\vec{n}=\overrightarrow{0}$. Since $D_{\vec{n}}=0$ whenever $n_{j}<0$ for some $j \in\{1, \ldots, r\}$ we find that $D_{\overrightarrow{0}} \leq A_{\overrightarrow{0}}$, which corresponds to the required result when $\vec{n}=\overrightarrow{0}$.

Suppose that the result is true for every multi-index of length $|\vec{n}|-1$. Then

$$
D_{\vec{n}} \leq A_{\vec{n}}+\sum_{j=1}^{r} a_{j} \sum_{\vec{m} \leq \vec{n}-\vec{e}_{j}} A_{\vec{n}-\vec{e}_{j}-\vec{m}}\left(\begin{array}{c}
|\vec{m}| \\
m_{1}, \ldots, m_{r}
\end{array}\right) a_{1}^{m_{1}} \cdots a_{r}^{m_{r}} .
$$

In the second sum we change the index $m_{j}$ to $m_{j}^{*}-1$ to find

$$
\sum_{\vec{m}^{*} \leq \vec{n}, m_{j}^{*} \neq 0} A_{\vec{n}-\vec{m}^{*}}\left(\begin{array}{c}
\left|\vec{m}^{*}\right|-1 \\
m_{1}, \ldots, m_{j}^{*}-1, \ldots, m_{r}
\end{array}\right) a_{1}^{m_{1}} \cdots a_{j}^{m_{j}^{*}-1} \cdots a_{r}^{m_{r}},
$$

where $\vec{m}^{*}=\left(m_{1}, \ldots, m_{j}^{*}, \ldots, m_{r}\right)$. Now use for $\left|\vec{m}^{*}\right| \neq 0$ the identity

$$
\left(\begin{array}{c}
\left|\vec{m}^{*}\right|-1 \\
m_{1}, \ldots, m_{j}^{*}-1, \ldots, m_{r}
\end{array}\right)=\frac{m_{j}^{*}}{\left|\vec{m}^{*}\right|}\left(\begin{array}{c}
\left|\vec{m}^{*}\right| \\
m_{1}, \ldots, m_{j}^{*}, \ldots, m_{r}
\end{array}\right)
$$

to find that the sum becomes

$$
\frac{1}{a_{j}\left|\vec{m}^{*}\right|} \sum_{\vec{m}^{*} \leq \vec{n}, m_{j}^{*} \neq 0} m_{j}^{*} A_{\vec{n}-\vec{m}^{*}}\left(\begin{array}{c}
\left|\vec{m}^{*}\right| \\
m_{1}, \ldots, m_{j}^{*}, \ldots, m_{r}
\end{array}\right) a_{1}^{m_{1}} \cdots a_{j}^{m_{j}^{*}} \cdots a_{r}^{m_{r}} .
$$

This gives

$$
D_{\vec{n}} \leq A_{\vec{n}}+\sum_{\overrightarrow{0} \neq \vec{m}^{*} \leq \vec{n}}\left(\sum_{j=1}^{r} \frac{m_{j}^{*}}{\left|\vec{m}^{*}\right|}\right) A_{\vec{n}-\vec{m}^{*}}\left(\begin{array}{c}
\left|\vec{m}^{*}\right| \\
m_{1}, \ldots, m_{r}
\end{array}\right) a_{1}^{m_{1}} \cdots a_{r}^{m_{r}},
$$

which immediately gives the required result.

Lemma 3.2. Suppose that $n_{j}=\left\lfloor q_{j} n\right\rfloor$ and $\sum_{j=1}^{r} q_{j}=1$ and that

$$
\lim _{n \rightarrow \infty} A_{\vec{n}}=0 \text {. }
$$

Then, if $0<a_{j}<a$, where $0<a<1 / r$ is such that $a^{q_{i}}<1 /(1+(r-1) a)$ for $1 \leq i \leq r$, one has

$$
\lim _{n \rightarrow \infty} \sum_{\vec{m} \leq \vec{n}} A_{\vec{n}-\vec{m}}\left(\begin{array}{c}
|\vec{m}| \\
m_{1}, \ldots, m_{r}
\end{array}\right) a_{1}^{m_{1}} \cdots a_{r}^{m_{r}}=0 .
$$

Proof. For every $\varepsilon>0$ there exists $n_{0} \in \mathbb{N}$ such that for all $n \geq n_{0}$ we have that $\left|A_{\vec{n}}\right|<\varepsilon$ whenever $n_{j} \geq\left\lfloor n_{0} q_{j}\right\rfloor$ for every $j \in\{1, \ldots, r\}$. We estimate the sum by

$$
\sum_{\vec{m} \leq \vec{n}}\left|A_{\vec{n}-\vec{m}}\right|\left(\begin{array}{c}
|\vec{m}| \\
m_{1}, \ldots, m_{r}
\end{array}\right) a_{1}^{m_{1}} \cdots a_{r}^{m_{r}} \leq \sum_{\vec{m} \in I_{0}} \cdots+\sum_{j=1}^{r} \sum_{\vec{m} \in I_{j}} \cdots
$$


where $I_{0}=\left\{\vec{m} \in \mathbb{N}^{r}: \vec{m} \leq \vec{n}-\vec{n}_{0}\right\}$ with $\vec{n}_{0}=\left(\left\lfloor n_{0} q_{1}\right\rfloor, \ldots,\left\lfloor n_{0} q_{r}\right\rfloor\right)$, and $I_{j}=\{\vec{m} \in$ $\left.\mathbb{N}^{r}: n_{j}-\left\lfloor n_{0} q_{j}\right\rfloor<m_{j} \leq n_{j}\right\}$. Note that the sets $I_{1}, \ldots, I_{r}$ are not disjoint so that we indeed get an upper bound. We have

$$
\begin{aligned}
\sum_{\vec{m} \in I_{0}}\left|A_{\vec{n}-\vec{m}}\right|\left(\begin{array}{c}
|\vec{m}| \\
m_{1}, \ldots, m_{r}
\end{array}\right) a_{1}^{m_{1}} \cdots a_{r}^{m_{r}} & \leq \varepsilon \sum_{\vec{m} \in I_{0}}\left(\begin{array}{c}
|\vec{m}| \\
m_{1}, \ldots, m_{r}
\end{array}\right) a_{1}^{m_{1}} \cdots a_{r}^{m_{r}} \\
& \leq \varepsilon \sum_{\vec{m} \leq \vec{n}}\left(\begin{array}{c}
|\vec{m}| \\
m_{1}, \ldots, m_{r}
\end{array}\right) a_{1}^{m_{1}} \cdots a_{r}^{m_{r}} \\
& =\varepsilon \sum_{k=0}^{|\vec{n}|}\left(\sum_{j=1}^{r} a_{j}\right)^{k} \\
& \leq \frac{\varepsilon}{1-r a},
\end{aligned}
$$

where we used that $a_{j}<a<1 / r$ for $1 \leq j \leq r$. On the other hand we know that the $A_{\vec{n}}$ are bounded, so that $\left|A_{\vec{n}}\right| \leq M$ for some $M>0$, and then

$$
\begin{aligned}
\sum_{\vec{m} \in I_{j}}\left|A_{\vec{n}-\vec{m}}\right|\left(\begin{array}{c}
|\vec{m}| \\
m_{1}, \ldots, m_{r}
\end{array}\right) & a_{1}^{m_{1}} \cdots a_{r}^{m_{r}} \\
& \leq M a_{j}^{n_{j}-\left\lfloor n_{0} q_{j}\right\rfloor} \sum_{\vec{m} \in I_{j}}\left(\begin{array}{c}
|\vec{m}| \\
m_{1}, \ldots, m_{r}
\end{array}\right) a_{1}^{m_{1}} \cdots 1 \cdots a_{r}^{m_{r}},
\end{aligned}
$$

where we have used $a_{j}^{m_{j}} \leq a_{j}^{n_{j}-\left\lfloor n_{0} q_{j}\right\rfloor}$, which holds on $I_{j}$. Furthermore we have

$$
\begin{gathered}
M a_{j}^{n_{j}-\left\lfloor n_{0} q_{j}\right\rfloor} \sum_{\vec{m} \in I_{j}}\left(\begin{array}{c}
|\vec{m}| \\
m_{1}, \ldots, m_{r}
\end{array}\right) a_{1}^{m_{1}} \cdots 1 \cdots a_{r}^{m_{r}} \\
\leq M a_{j}^{n_{j}-\left\lfloor n_{0} q_{j}\right\rfloor} \sum_{\vec{m} \leq \vec{n}}\left(\begin{array}{c}
|\vec{m}| \\
m_{1}, \ldots, m_{r}
\end{array}\right) a_{1}^{m_{1}} \cdots 1 \cdots a_{r}^{m_{r}} \\
=M a_{j}^{n_{j}-\left\lfloor n_{0} q_{j}\right\rfloor} \sum_{k=0}^{|\vec{n}|}\left(\begin{array}{c}
1+\sum_{i=1, i \neq j}^{r} a_{i}
\end{array}\right)^{k} \\
\leq M a^{n_{j}-\left\lfloor n_{0} q_{j}\right\rfloor} \frac{(1+(r-1) a)^{|\vec{n}|+1}-1}{(r-1) a},
\end{gathered}
$$

where we used that $a_{j} \leq a$. Clearly,

$$
\lim _{n \rightarrow \infty} a^{n q_{j}}(1+(r-1) a)^{n}=0
$$

whenever $a^{q_{j}}(1+(r-1) a)<1$, and this is indeed what was assumed to be true for a. Combining our estimates we have

$$
\limsup _{n \rightarrow \infty}\left|\sum_{\vec{m} \leq \vec{n}} A_{\vec{n}-\vec{m}}\left(\begin{array}{c}
|\vec{m}| \\
m_{1}, \ldots, m_{r}
\end{array}\right) a_{1}^{m_{1}} \cdots a_{r}^{m_{r}}\right| \leq \frac{\varepsilon}{1-r a},
$$

and since this holds for every $\varepsilon>0$, the required result follows. 
Lemma 3.3. Suppose that (1.2) holds, where $n_{j}=\left\lfloor q_{j} n\right\rfloor$, with $q_{j}>0$ and $\sum_{j=1}^{r} q_{j}=1$. Then we have, uniformly on compact subsets $K \subset \mathbb{C} \backslash \mathbb{R}$,

$$
\lim _{n \rightarrow \infty}\left|\frac{P_{\vec{n}}(x)}{P_{\vec{n}+\vec{e}_{k}}(x)}-\frac{P_{\vec{n}-\vec{e}_{\ell}}(x)}{P_{\vec{n}+\vec{e}_{k}-\vec{e}_{\ell}}(x)}\right|=0,
$$

for any $k$ and $\ell$ in $\{1, \ldots, r\}$.

Proof. From the recurrence relation (1.1) we have

$$
x=\frac{P_{\vec{n}+\vec{e}_{k}}(x)}{P_{\vec{n}}(x)}+b_{\vec{n}, k}+\sum_{j=1}^{r} a_{\vec{n}, j} \frac{P_{\vec{n}-\vec{e}_{j}}(x)}{P_{\vec{n}}(x)} .
$$

The same relation but with $\vec{n}$ replaced by $\vec{n}-\vec{e}_{\ell}$ gives

$$
x=\frac{P_{\vec{n}+\vec{e}_{k}-\vec{e}_{\ell}}(x)}{P_{\vec{n}-\vec{e}_{\ell}}(x)}+b_{\vec{n}-\vec{e}_{\ell}, k}+\sum_{j=1}^{r} a_{\vec{n}-\vec{e}_{\ell}, j} \frac{P_{\vec{n}-\vec{e}_{j}-\vec{e}_{\ell}}(x)}{P_{\vec{n}-\vec{e}_{\ell}}(x)} .
$$

Subtract both equations to find

$$
\begin{aligned}
\frac{P_{\vec{n}+\vec{e}_{k}}(x)}{P_{\vec{n}}(x)}-\frac{P_{\vec{n}+\vec{e}_{k}-\vec{e}_{\ell}}(x)}{P_{\vec{n}-\vec{e}_{\ell}}(x)}= & b_{\vec{n}-\vec{e}_{\ell}, k}-b_{\vec{n}, k}-\sum_{j=1}^{r} a_{\vec{n}, j}\left(\frac{P_{\vec{n}}-\vec{e}_{j}(x)}{P_{\vec{n}}(x)}-\frac{P_{\vec{n}-\vec{e}_{j}-\vec{e}_{\ell}}(x)}{P_{\vec{n}-\vec{e}_{\ell}}(x)}\right) \\
& -\sum_{j=1}^{r}\left(a_{\vec{n}, j}-a_{\vec{n}-\vec{e}_{\ell}, j}\right) \frac{P_{\vec{n}-\vec{e}_{j}-\vec{e}_{\ell}}(x)}{P_{\vec{n}-\vec{e}_{\ell}}(x)} .
\end{aligned}
$$

If we use the bound (2.2), then

$$
\left|\frac{P_{\vec{n}+\vec{e}_{k}}(x)}{P_{\vec{n}}(x)}-\frac{P_{\vec{n}+\vec{e}_{k}-\vec{e}_{\ell}}(x)}{P_{\vec{n}-\vec{e}_{\ell}}(x)}\right| \geq \delta^{2}\left|\frac{P_{\vec{n}}(x)}{P_{\vec{n}+\vec{e}_{k}}(x)}-\frac{P_{\vec{n}-\vec{e}_{\ell}}(x)}{P_{\vec{n}+\vec{e}_{k}-\vec{e}_{\ell}}(x)}\right|
$$

so that

$$
\begin{aligned}
\left|\frac{P_{\vec{n}}(x)}{P_{\vec{n}+\vec{e}_{k}}(x)}-\frac{P_{\vec{n}-\vec{e}_{\ell}}(x)}{P_{\vec{n}+\vec{e}_{k}-\vec{e}_{\ell}}(x)}\right| \leq & \frac{1}{\delta^{2}} \sum_{j=1}^{r}\left|a_{\vec{n}, j}\right|\left|\frac{P_{\vec{n}-\vec{e}_{j}}(x)}{P_{\vec{n}}(x)}-\frac{P_{\vec{n}-\vec{e}_{j}-\vec{e}_{\ell}}(x)}{P_{\vec{n}-\vec{e}_{\ell}}(x)}\right| \\
& +\frac{1}{\delta^{2}}\left|b_{\vec{n}, k}-b_{\vec{n}-\vec{e}_{\ell}, k}\right|+\frac{1}{\delta^{3}} \sum_{j=1}^{r}\left|a_{\vec{n}, j}-a_{\vec{n}-\vec{e}_{\ell}, j}\right| .
\end{aligned}
$$

If we use the notation

$$
D_{\vec{n}, k, \ell}=\left|\frac{P_{\vec{n}}(x)}{P_{\vec{n}+\vec{e}_{k}}(x)}-\frac{P_{\vec{n}-\vec{e}_{\ell}}(x)}{P_{\vec{n}+\vec{e}_{k}-\vec{e}_{\ell}}(x)}\right|,
$$

then this gives

$$
D_{\vec{n}, k, \ell} \leq \frac{1}{\delta^{2}}\left|b_{\vec{n}, k}-b_{\vec{n}-\vec{e}_{\ell}, k}\right|+\frac{1}{\delta^{3}} \sum_{j=1}^{r}\left|a_{\vec{n}, j}-a_{\vec{n}-\vec{e}_{\ell}, j}\right|+\frac{1}{\delta^{2}} \sum_{j=1}^{r}\left|a_{\vec{n}, j}\right| D_{\vec{n}-\vec{e}_{j}, j, \ell} .
$$

The convergence (1.2) implies that $\left|a_{\vec{n}, j}\right| \leq \hat{a}_{j}$ for certain constants $\hat{a}_{1}, \ldots, \hat{a}_{r}$. Now denote

$$
D_{\vec{n}, \ell}=\max _{1 \leq k \leq r} D_{\vec{n}, k, \ell},
$$

then we arrive at the inequality

$$
D_{\vec{n}, \ell} \leq \sum_{j=1}^{r}\left(\frac{\left|b_{\vec{n}, j}-b_{\vec{n}-\vec{e}_{\ell}, j}\right|}{\delta^{2}}+\frac{\left|a_{\vec{n}, j}-a_{\vec{n}-\vec{e}_{\ell}, j}\right|}{\delta^{3}}\right)+\sum_{j=1}^{r} \frac{\hat{a}_{j}}{\delta^{2}} D_{\vec{n}-\vec{e}_{j}, \ell} .
$$


From Lemma 3.1 we then find

$$
D_{\vec{n}, \ell} \leq \sum_{\vec{m} \leq \vec{n}} A_{\vec{n}-\vec{m}}\left(\begin{array}{c}
|\vec{m}| \\
m_{1}, \ldots, m_{r}
\end{array}\right) \frac{\hat{a}_{1}^{m_{1}} \cdots \hat{a}_{r}^{m_{r}}}{\delta^{2|\vec{m}|}},
$$

where

$$
A_{\vec{n}}=\sum_{j=1}^{r}\left(\frac{\left|b_{\vec{n}, j}-b_{\vec{n}-\vec{e}_{\ell}, j}\right|}{\delta^{2}}+\frac{\left|a_{\vec{n}, j}-a_{\vec{n}-\vec{e}_{\ell}, j}\right|}{\delta^{3}}\right) .
$$

If we write

$$
a_{\vec{n}, j}=a_{n, j}\left(q_{1}, \ldots, q_{r}\right), \quad b_{\vec{n}, j}=b_{n, j}\left(q_{1}, \ldots, q_{r}\right),
$$

then

$$
a_{\vec{n}-\vec{e}_{\ell}, j}=a_{n, j}\left(q_{1}, \ldots, q_{\ell}-\frac{1}{n}, \ldots, q_{r}\right), \quad b_{\vec{n}-\vec{e}_{\ell}, j}=b_{n, j}\left(q_{1}, \ldots, q_{\ell}-\frac{1}{n}, \ldots, q_{r}\right),
$$

and our assumption that (1.2) holds uniformly in a closed neighborhood of $\vec{q}$ then implies that

$$
\lim _{n \rightarrow \infty} a_{\vec{n}-\vec{e}_{\ell}, j}=\lim _{n \rightarrow \infty} a_{\vec{n}, j}=a_{j}, \quad \lim _{n \rightarrow \infty} a_{\vec{n}-\vec{e}_{\ell}, j}=\lim _{n \rightarrow \infty} a_{\vec{n}, j}=a_{j} .
$$

Hence for every $\delta>0$ we have that $A_{\vec{n}} \rightarrow 0$ as $n \rightarrow \infty$, where $n_{j}=\left\lfloor n q_{j}\right\rfloor$. If we choose the compact set $K^{*}$ such that $\delta$ is large enough, then $\hat{a}_{j} / \delta^{2}$ can be made sufficiently small so that Lemma 3.2 can be applied, from which we find that $D_{\vec{n}, \ell} \rightarrow 0$ uniformly for $x \in K^{*}$. Note that $D_{\vec{n}, \ell} \leq 2 \delta$ on any compact $K \subset \mathbb{C} \backslash \mathbb{R}$, hence by Vitali's theorem [12, Thm. $12.8 \mathrm{~d}$ on p. 566] we may conclude that $D_{\vec{n}, \ell}$ converges uniformly to 0 on every compact set $K \subset \mathbb{C} \backslash \mathbb{R}$. This is what needed to be proved.

\section{Proof of Theorem 1.2}

The proof of Theorem 1.2 is very similar to the proof of Theorem 1.1. We only give the modifications which are needed. Use the recurrence relation (1.1) but with $x$ replaced by $n^{\gamma} x$, and divide by $P_{\vec{n}}\left(n^{\gamma} x\right)$ to find

$$
x=\frac{P_{\vec{n}+\vec{e}_{k}}\left(n^{\gamma} x\right)}{n^{\gamma} P_{\vec{n}}\left(n^{\gamma} x\right)}+\frac{b_{\vec{n}, k}}{n^{\gamma}}+\sum_{j=1}^{r} \frac{a_{\vec{n}, j}}{n^{2 \gamma}} \frac{n^{\gamma} P_{\vec{n}-\vec{e}_{j}}\left(n^{\gamma} x\right)}{P_{\vec{n}}\left(n^{\gamma} x\right)} .
$$

The partial fractions decomposition now is

$$
\frac{n^{\gamma} P_{\vec{n}-\vec{e}_{j}}\left(n^{\gamma} x\right)}{P_{\vec{n}}\left(n^{\gamma} x\right)}=\sum_{k=1}^{|\vec{n}|} \frac{A_{\vec{n}, k}}{x-x_{\vec{n}, k} / n^{\gamma}},
$$

and we have the bound

$$
\left|\frac{n^{\gamma} P_{\vec{n}-\vec{e}_{j}}\left(n^{\gamma} x\right)}{P_{\vec{n}}\left(n^{\gamma} x\right)}\right| \leq \sum_{k=1}^{|\vec{n}|} \frac{A_{\vec{n}, k}}{\left|x-x_{\vec{n}, k} / n^{\gamma}\right|} \leq \frac{1}{\delta},
$$

where $\delta=\min \{|x-y|: x \in K, y \in \mathbb{R}\}>0$ is the minimal distance between $K$ and $\mathbb{R}$. Montel's theorem gives a subsequence $\left(n_{k}\right)_{k \in \mathbb{N}}$ such that

$$
\lim _{k \rightarrow \infty} \frac{n_{k}^{\gamma} P_{\vec{n}_{k}-\vec{e}_{j}}\left(n_{k}^{\gamma} x\right)}{P_{\vec{n}_{k}}\left(n_{k}^{\gamma} x\right)}=h_{j}(x),
$$

uniformly on $K$ for every $j$ with $1 \leq j \leq r$, where $h_{j}$ is analytic on $K$. From then on the proof of Theorem 1.1 can be repeated, except that one uses (4.1) and (1.5) 
instead of (2.1) and (1.2). One finally ends up with the algebraic equation (1.6) which is in fact the same equation as in (1.3).

\section{Examples}

5.1. Jacobi-Piñeiro polynomials. These are multiple orthogonal for the Jacobi weights $d \mu_{j}(x)=x^{\alpha_{j}}(1-x)^{\beta} d x$ on $[0,1]$, where $\alpha_{j}, \beta>-1$ and $\alpha_{i}-\alpha_{j} \notin \mathbb{Z}$. The recurrence coefficients are given by

$$
\begin{aligned}
a_{\vec{n}, j}=\frac{n_{j}\left(n_{j}+\alpha_{j}\right)(|\vec{n}|+\beta)}{\left(|\vec{n}|+n_{j}+\alpha_{j}+\beta+1\right)\left(|\vec{n}|+n_{j}+\alpha_{j}+\beta\right)\left(|\vec{n}|+n_{j}+\alpha_{j}+\beta-1\right)} \\
\quad \times \prod_{i=1}^{r} \frac{|\vec{n}|+\alpha_{i}+\beta}{|\vec{n}|+n_{i}+\alpha_{i}+\beta} \prod_{i \neq j} \frac{n_{j}+\alpha_{j}-\alpha_{i}}{n_{j}-n_{i}+\alpha_{j}-\alpha_{i}},
\end{aligned}
$$

and a more complicated expression for $b_{\vec{n}, j}($ see $[\mathbf{1 7}, \S 5.5])$. We therefore have

$$
\lim _{n \rightarrow \infty} a_{\vec{n}, j}=\frac{q_{j}^{r+1}}{\left(1+q_{j}\right)^{3}} \prod_{i=1}^{r} \frac{1}{1+q_{i}} \prod_{i \neq j} \frac{1}{q_{j}-q_{i}} .
$$

This means that the nearest neighbor recurrence coefficients $a_{\vec{n}, j}$ converge only when $q_{i} \neq q_{j}$ for every $i \neq j$. Note that the $a_{\vec{n}, j}$ are not necessarily positive, but it is known that the zeros of $P_{\vec{n}}$ and $P_{\vec{n}+\vec{e}_{k}}$ interlace since the measures $\left(\mu_{1}, \ldots, \mu_{r}\right)$ form an AT system (see [11, Thm. 2.1]).

5.2. Multiple Hermite polynomials. They satisfy

$$
\int_{-\infty}^{\infty} H_{\vec{n}}(x) x^{k} e^{-x^{2}+c_{j} x} d x=0, \quad k=0,1, \ldots, n_{j}-1,1 \leq j \leq r,
$$

where $c_{j} \in \mathbb{R}$ and $c_{i} \neq c_{j}$ whenever $i \neq j$. The nearest neighbor recurrence relations are

$$
x H_{\vec{n}}(x)=H_{\vec{n}+\vec{e}_{k}}(x)+\frac{c_{k}}{2} H_{\vec{n}}(x)+\sum_{j=1}^{r} \frac{n_{j}}{2} H_{\vec{n}-\vec{e}_{j}}(x),
$$

$[\mathbf{1 3}, \S 23.5]$ so that $a_{\vec{n}, j}=n_{j} / 2$ and $b_{\vec{n}, k}=c_{k} / 2$. This means that we have to use the scaling with $\gamma=1 / 2$ to find

$$
\lim _{n \rightarrow \infty} \frac{a_{\vec{n}, j}}{n}=\frac{q_{j}}{2}, \quad \lim _{n \rightarrow \infty} \frac{b_{\vec{n}, j}}{\sqrt{n}}=0 .
$$

This is not very convenient since this implies that the limiting values $b_{1}, \ldots, b_{r}$ all coincide and $B_{r}$ hence has a zero of multiplicity $r$. We can't use the partial fraction decomposition (1.7) and hence we cannot determine the polynomial $A_{r-1}$. It is more interesting to let the parameters depend on $n$ and to consider $b_{\vec{n}, j}=c_{j} \sqrt{n}$, so that

$$
\lim _{n \rightarrow \infty} \frac{b_{\vec{n}, j}}{\sqrt{n}}=c_{j} / 2
$$

This happens to be the case of interest when one is dealing with random matrices with external source $[\mathbf{1}, \mathbf{5}, \mathbf{6}, \mathbf{7}]$ and non-intersecting Brownian motions leaving from $r$ different points and arriving at one point $[\mathbf{8}, \mathbf{9}]$. 
5.3. Multiple Laguerre polynomials. There are two kinds of multiple Laguerre polynomials $[\mathbf{1 3}, \S 23.4]$. The polynomials of the first kind satisfy

$$
\int_{0}^{\infty} L_{\vec{n}}(x) x^{k} x^{\alpha_{j}} e^{-x} d x=0, \quad k=0,1, \ldots, n_{j}-1,1 \leq j \leq r,
$$

where $\alpha_{1}, \ldots, \alpha_{r}>-1$ and $\alpha_{i}-\alpha_{j} \notin \mathbb{Z}$, and the recurrence coefficients are $[\mathbf{1 7}$, $\S 5.3]$

$$
a_{\vec{n}, j}=n_{j}\left(n_{j}+\alpha_{j}\right) \prod_{i \neq j} \frac{n_{j}+\alpha_{j}-\alpha_{i}}{n_{j}-n_{i}+\alpha_{j}-\alpha_{i}}, \quad b_{\vec{n}, j}=|\vec{n}|+n_{j}+\alpha_{j}+1 .
$$

We can use Theorem 1.2 with the scaling $\gamma=1$ and find

$$
\lim _{n \rightarrow \infty} \frac{a_{\vec{n}, j}}{n^{2}}=q_{j}^{r+1} \prod_{i \neq j} \frac{1}{q_{j}-q_{i}}, \quad \lim _{n \rightarrow \infty} \frac{b_{\vec{n}, j}}{n}=1+q_{j} .
$$

Observe that the limit for $a_{\vec{n}, j} / n^{2}$ does not exist when $q_{j}=q_{i}$ for some $i$, and in that case the limit of $b_{\vec{n}, j} / n$ and $b_{\vec{n}, i} / n$ is the same, so that the partial fraction decomposition (1.7) is not possible. So our theorem can only be used when $q_{i} \neq q_{j}$ whenever $i \neq j$.

Multiple Laguerre polynomials of the second kind satisfy

$$
\int_{0}^{\infty} L_{\vec{n}}(x) x^{k} x^{\alpha} e^{-c_{j} x} d x=0, \quad k=0,1, \ldots, n_{j}-1,1 \leq j \leq r,
$$

where $\alpha>-1$ and $c_{1}, \ldots, c_{r}>0$ with $c_{i} \neq c_{j}$ whenever $i \neq j$. The recurrence coefficients are $[\mathbf{1 7}, \S 5.3]$

$$
a_{\vec{n}, j}=\frac{n_{j}}{c_{j}^{2}}(|\vec{n}|+\alpha), \quad b_{\vec{n}, j}=\frac{|\vec{n}|+\alpha+1}{c_{j}}+\sum_{i=1}^{r} \frac{n_{i}}{c_{i}},
$$

so that again we need the scaling $\gamma=1$. Observe that all the $a_{\vec{n}, j}$ are now strictly positive whenever $n_{j}>0$ so that we have interlacing of the zeros of neighboring polynomials. Furthermore, we have

$$
\lim _{n \rightarrow \infty} \frac{a_{\vec{n}, j}}{n^{2}}=\frac{q_{j}}{c_{j}^{2}}, \quad \lim _{n \rightarrow \infty} \frac{b_{\vec{n}, j}}{n}=\frac{1}{c_{j}}+\sum_{i=1}^{r} \frac{q_{i}}{c_{i}},
$$

so that all the limits $b_{1}, \ldots, b_{r}$ are different.

5.4. Multiple Charlier polynomials. These are discrete multiple orthogonal polynomials satisfying

$$
\sum_{k=0}^{\infty} C_{\vec{n}}(k) k^{\ell} \frac{a_{j}^{k}}{k !}=0, \quad \ell=0,1, \ldots, n_{j}-1,1 \leq j \leq r,
$$

where $a_{1}, \ldots, a_{r}>0$ and $a_{i} \neq a_{j}$ whenever $i \neq j[\mathbf{1 3}, \S 23.6 .1]$. The nearest neighbor recurrence relations are

$$
x C_{\vec{n}}(x)=C_{\vec{n}+\vec{e}_{k}}(x)+\left(a_{k}+|\vec{n}|\right) C_{\vec{n}}(x)+\sum_{j=1}^{r} a_{j} n_{j} C_{\vec{n}-\vec{e}_{j}}(x),
$$

so that $a_{\vec{n}, j}=a_{j} n_{j}$ and $b_{\vec{n}, j}=a_{j}+|\vec{n}|$. Again the $a_{\vec{n}, j}$ are positive whenever $n_{j}>0$. We need to use a scaling with $\gamma=1$ and to scale $a_{k}$ to $a_{k} n$ to get different limits $b_{1}, \ldots, b_{r}$. This case was worked out earlier in [16]. 


\section{Concluding remarks}

Why is it useful to investigate the ratio asymptotics for multiple orthogonal polynomials? Naturally, it is an extension of the analysis of ratio asymptotic of orthogonal polynomials on the real line, which is connected with Nevai's class $M(a, b)$ and Rakhmanov's theorem. Apart from that, there are three more explicit reasons

(1) The ratio asymptotics allows to find the asymptotic zero distribution of the multiple orthogonal polynomials. Indeed, if $x_{\vec{n}, i}(1 \leq i \leq|\vec{n}|)$ are the zeros of $P_{\vec{n}}$, then

$$
\lim _{n \rightarrow \infty} \frac{1}{|\vec{n}|} \sum_{i=1}^{|\vec{n}|} f\left(x_{\vec{n}, i}\right)=\int f(t) d \nu(t)
$$

for every bounded and continuous function $f$ on $\mathbb{R}$, where the Stieltjes transform of the measure $\nu$ is given by

$$
\int \frac{d \nu(t)}{x-t}=\lim _{n \rightarrow \infty} \frac{1}{|\vec{n}|} \frac{P_{\vec{n}}^{\prime}(x)}{P_{\vec{n}}(x)} .
$$

A slight modification is needed when one uses the a scaling $n^{\gamma}$ as in Theorem 1.2. The asymptotic zero distribution can therefore be obtained from the ratio asymptotics through the formula

$$
\frac{P_{\vec{n}}^{\prime}(x)}{P_{\vec{n}}(x)}=\frac{P_{\vec{n}-n_{r} \vec{e}_{r}}^{\prime}(x)}{P_{\vec{n}-n_{r} \vec{e}_{r}}(x)}+\sum_{k=0}^{n_{r}-1}\left(\frac{P_{\vec{n}-k \vec{e}_{r}}(x)}{P_{\vec{n}-(k+1) \vec{e}_{r}}(x)}\right)^{\prime} /\left(\frac{P_{\vec{n}-k \vec{e}_{r}}(x)}{P_{\vec{n}-(k+1) \vec{e}_{r}}(x)}\right),
$$

which expresses the ratio $P_{\vec{n}}^{\prime}(x) / P_{\vec{n}}(x)$ for $r$ measures as a similar ratio for $r-1$ measures and the ratio of neighboring multiple orthogonal polynomials, for which the asymptotic behavior is given in Theorems 1.1 and 1.2 .

(2) It is known that there usually is a vector equilibrium problem of the form: minimize the energy functional

$$
E\left(\nu_{1}, \ldots, \nu_{r}\right)=\sum_{i=1}^{r} \sum_{j=1}^{r} C_{i, j} I\left(\nu_{i}, \nu_{j}\right)+\sum_{j=1}^{r} \int V_{j}(x) d \nu_{j}(x)
$$

where $I\left(\nu_{i}, \nu_{j}\right)$ is the mutual logarithmic energy of $\nu_{i}$ and $\nu_{j}$ and $V_{j}$ is an external field, possibly with constraints $\nu_{i} \leq \sigma_{i}$ for some $1 \leq i \leq r$. To find the solution, one often needs a Riemann surface and certain rational functions on that Riemann surface. The Riemann surface for the algebraic function $z$ satisfying (1.3) is the natural geometric object in this case. This Riemann surface has $r+1$ sheets, it has genus 0 and there are branch points when (1.3) has multiple roots, i.e., when $B_{r}^{2}(z)-A_{r-1}(z) B_{r}^{\prime}(z)+$ $A_{r-1}^{\prime}(z) B_{r}(z)=0$, which shows that there are $2 r$ branch points.

(3) If one wants to investigate the (strong) asymptotic behavior of the multiple orthogonal polynomials, then a useful method is to use the RiemannHilbert problem for the multiple orthogonal polynomials [18]. If one wants to use the Deift-Zhou steepest descent analysis for Riemann-Hilbert problems, then the first step is to transform the Riemann-Hilbert problem to a Riemann-Hilbert problem which is normalized at infinity. This requires knowledge of the (expected) asymptotic behavior of $P_{\vec{n}}(x)$, which can be expressed in terms of the measures $\left(\nu_{1}, \ldots, \nu_{r}\right)$ from the equilibrium 
problem. Again the Riemann surface for (1.3) is the natural object for the Riemann-Hilbert analysis.

Our Theorems 1.1 and 1.2 have to be modified in case some of the limits $b_{1}, \ldots, b_{r}$ are equal, which is the case for multiple Laguerre polynomials of the first kind and Jacobi-Piñeiro polynomials when $q_{i}=q_{j}$ for some $i \neq j$. If $b_{1}^{*}, \ldots, b_{s}^{*}$ are the distinct limits, then one has to use the polynomial $B_{s}(z)=\left(z-b_{1}^{*}\right) \cdots\left(z-b_{s}^{*}\right)$ of degree $s<r$. Then (2.3) implies that

$$
x-z=\sum_{j=1}^{s} \frac{a_{j}^{*}}{z-b_{j}^{*}}, \quad a_{j}^{*}=\sum_{b_{i}=b_{j}^{*}} a_{i} .
$$

So one really needs the asymptotic behavior of the sum $\sum_{b_{i}=b_{j}^{*}} a_{\vec{n}, i}$ rather than the behavior of every individual $a_{\vec{n}, j}$ in this case.

\section{References}

1. A.I Aptekarev, P.M. Bleher, A.B.J. Kuijlaars, Large $n$ limit of Gaussian random matrices with external source. II, Comm. Math. Phys. 259 (2005), no. 2, 367-389.

2. A.I. Aptekarev, V. Kalyagin, G. López Lagomasino, I.A. Rocha, On the limit behavior of recurrence coefficients for multiple orthogonal polynomials, J. Approx. Theory 139 (2006), no. $1-2,346-370$.

3. A.I. Aptekarev, V.A. Kalyagin, E.B. Saff, E. B., Higher-order three-term recurrences and asymptotics of multiple orthogonal polynomials, Constr. Approx. 30 (2009), no. 2, 175-223.

4. A.I. Aptekarev, G. López Lagomasino, I.A. Rocha, Asymptotic behavior of the ratio of Hermite-Padé polynomials for Nikishin systems (in Russian), Mat. Sb. 196 (2005), no. 8, 3-20; translation in Sb. Math. 196 (2005), no. 7-8, 1089-1107.

5. P.M. Bleher, A.B.J Kuijlaars, Random matrices with external source and multiple orthogonal polynomials, Int. Math. Res. Not. 2004 (2004), no. 3, 109-129.

6. P. Bleher, A.B.J. Kuijlaars, Large $n$ limit of Gaussian random matrices with external source. I, Comm. Math. Phys. 252 (2004), no. 1-3, 43-76.

7. P.M. Bleher, A.B.J. Kuijlaars, Large $n$ limit of Gaussian random matrices with external source. III. Double scaling limit, Comm. Math. Phys. 270 (2007), no. 2, 481-517.

8. E. Daems, A.B.J. Kuijlaars, Multiple orthogonal polynomials of mixed type and nonintersecting Brownian motions, J. Approx. Theory 146 (2007), no. 1, 91-114.

9. E. Daems, A.B.J. Kuijlaars, W. Veys, Asymptotics of non-intersecting Brownian motions and a $4 \times 4$ Riemann-Hilbert problem, J. Approx. Theory 153 (2008), no. 2, 225-256.

10. S. Delvaux, A. López, G. López Lagomasino, On a family of Nikishin systems with periodic recurrence coefficients (Russian), Mat. Sb. 204 (2013), no. 1, 47-78; translation in Sb. Math. 204 (2013), no. 1-2, 43-74.

11. M. Haneczok, W. Van Assche, Interlacing properties of zeros of multiple orthogonal polynomials, J. Math. Anal. Appl. 389 (2012), 429-438.

12. P. Henrici, Applied and Computational Complex Analysis, Vol. 2, Wiley Classics Library, John Wiley \& Sons, New York, 1991. Original published in 1977.

13. M.E.H. Ismail, Classical and Quantum Orthogonal Polynomials in One Variable, Encyclopedia of Mathematics and its Applications 98, Cambridge University Press, 2005 (paperback edition, 2009).

14. V.A. Kaliaguine, On operators associated with Angelesco systems, East J. Approx. 1 (1995), no. $2,157-170$.

15. A. López García, G. López Lagomasino, Ratio asymptotic of Hermite-Padé orthogonal polynomials for Nikishin systems. II, Adv. Math. 218 (2008), no. 4, 1081-1106.

16. F. Ndayiragije, W. Van Assche, Asymptotics for the ratio and the zeros of multiple Charlier polynomials, J. Approx. Theory 164 (2012), 823-840.

17. W. Van Assche, Nearest neighbor recurrence relations for multiple orthogonal polynomials, J. Approx. Theory 163 (2011), 1427-1448.

18. W. Van Assche, J.S. Geronimo, A.B.J. Kuijlaars, Riemann-Hilbert problems for multiple orthogonal polynomials, in "Special functions 2000: current perspective and future directions" 
(Tempe, AZ), NATO Sci. Ser. II Math. Phys. Chem. 30, Kluwer Acad. Publ., Dordrecht, 2001, pp. 23-59.

KU Leuven, Department of Mathematics, Celestijnenlaan 200 B box 2400, BE-3001 Leuven, Belgium

E-mail address: walter@wis.kuleuven.be 